\title{
Correlative in situ Analysis of Magnetosome Magnetite Biomineralization
}

Taylor J. Woehl ${ }^{1}$, Emre Firlar ${ }^{1}$, Teresa Perez-Gonzalez ${ }^{2}$, Agatha Olszewska ${ }^{2}$, Damien Faivre ${ }^{2}$ and Tanya Prozorov ${ }^{1}$

${ }^{1 .}$ Emergent Atomic and Magnetic Structures, Division of Materials Sciences and Engineering, US DOE Ames Laboratory, Ames, IA 50011, U.S.A.

2. Department of Biomaterials, Max Planck Institute of Colloids and Interfaces, Science Park Golm, 14424 Potsdam, Germany.

Magnetotactic bacteria are widely used as a model system for the study of molecular mechanisms of magnetite magnetosome biomineralization. These microorganisms form magnetite nanocrystals with consistent species-specific morphologies, nearly perfect mineral crystal structures and well-defined magnetic properties. Bacterial magnetite biomineralization is a complex process involving a number of simultaneously occurring different steps, and understanding magnetosome magnetite biomineralization is of fundamental interest to devising the strategies for bioinspired synthesis of magnetic materials at the nanoscale [1]. Exploring the new analytical approaches aimed at specimen characterization in situ opens up new ways for the study of dynamics of process at the single-cell level and the nanometer-scale resolution. As many metabolic processes, including iron uptake by the bacteria, occur concurrently with the magnetite crystal formation and growth within the microorganism, development of powerful correlative optical and electron microscopy techniques suitable for accessing the cellular function and ultrastructure with necessary resolution, are crucial in understanding both types of processes.

Current studies point to a number of steps involved in magnetosome growth, including cellular uptake of soluble iron, its complexation with membrane proteins, and nucleation and growth of a mature magnetosome. However, the molecular mechanism of biomineralization in magnetotactic bacteria and the magnetosome nucleation and growth process remains unclear. Magnetosome magnetite biomineralization in Magnetospirillum magneticum strain AMB-1 was probed in situ by using a continuous flow fluid cell scanning transmission electron microscopy (S/TEM) holder platform in combination with the conventional electron microscopy imaging and analytical spectroscopy approaches. The bacteria were studied using a high angle annular dark field (HAADF) detector, where upon the biomineralization of iron, the high atomic number of magnetosomes chains in the bacteria provided high contrast allowing imaging of the microorganisms. STEM visualization in situ was followed by correlative fluorescence imaging to probe the integrity of bacterial cell wall membrane and confirm the bacteria's viability, as schematically shown in Figure 1. A fluorescent dye mixture was flowed through the fluid cell. As a result, the microorganisms with intact cell wall membrane were stained with a green fluorescent protein and considered viable. Bacterial cells with damaged cell wall membrane were stained red with propidium iodide and considered dead [2]. We discuss the effects of the electron beam and confinement in the fluid cell and outline the steps to mitigate electron beaminduced damage to the bacterial cells.

While the crystal structure of magnetosome magnetite is well-established, far less is known about the chemical environment of the biomineral at the early stages of magnetosome formation. Current effort is focused on probing the magnetosome magnetite biomineralization both in vivo and in vitro by using the combination of correlative STEM-FM imaging, electron diffraction, and analytical spectroscopy. To this end, we employed the combination of electron diffraction and EELS analyses to probe the crystallinity 
and chemical bonding in the nascent magnetosome particles. Newly formed magnetosome particles are mostly amorphous, whereas the fully grown magnetosomes are unambiguously indexed to magnetite. We have identified the narrow "transition range" corresponding to the onset of crystal lattice formation and utilized the peak fitting analysis of EEL spectra to monitor the evolution of chemical bonding in magnetosomes and correlated these findings with the emergence of crystalline lattice [3].

In-vivo imaging of viable bacteria is a first step in directly observing biomineralization of magnetosomes in live magnetotactic bacteria. Our approach can be expanded to the in vivo characterization of a wide range of inorganic structures biomineralized by various microorganisms, and as such it is expected to have a direct impact on the understanding of biological processes. Assuming the radiation damage to the specimen is mitigated, additional characterization will include X-ray fluorescence study of magnetotactic bacteria in the fluid cell for monitoring the cellular dynamics in situ. These studies will complement the in situ HAADF imaging effort, as shown in Figure 2 [4].

\section{References:}

[1] T Prozorov et al, Mater Sci Eng R 173 (2013), p. 133.

[2] TJ Woehl et al, Sci Rep 4 (2014), p. 6854.

[3] E Firlar et al, J Mater Res 31 (2016), p. 547.

[4] This work was supported by the U.S. Department of Energy (DOE), Office of Science, Basic Energy Sciences, Division of Materials Sciences and Engineering. T.P. acknowledges support from the Department of Energy Office of Science Early Career Research Award, Biomolecular Materials Program. The research was performed at the Ames Laboratory, which is operated for the U.S. Department of Energy by Iowa State University under Contract No. DE-AC02-07CH11358.

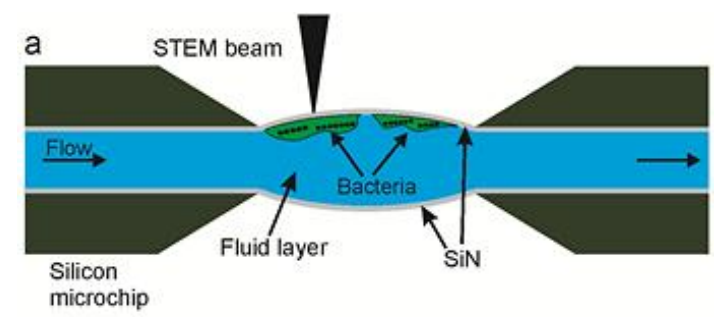

Figure 1. Schematics of HAADF-STEM imaging of magnetotactic bacteria in the Fluid Cell.

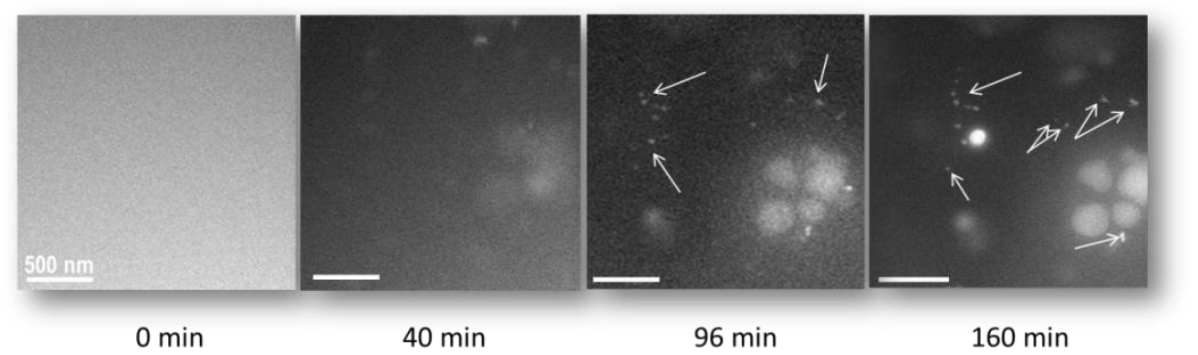

Figure 2. In situ magnetosome magnetite biomineralization by AMB-1 grown under low-iron conditions is observed after delivering iron-containing growth medium to the fluid cell. The continuous change in contrast is related to accumulation of iron preceding the magnetosome formation. Arrows point to formed magnetosomes. 